\title{
Broadband Dielectric Resonator Antenna With an Offset Well
}

\author{
Tze-Hsuan Chang, Student Member, IEEE, and Jean-Fu Kiang, Member, IEEE
}

\begin{abstract}
A broadband dielectric resonator (DR) antenna with an offset well is proposed. The well perturbs the original field distribution in the DR block to increase the impedance bandwidth associated with the $\mathrm{TE}_{111}^{y}$ mode. The feeding aperture generates a similar radiation pattern at adjacent band. The overall bandwidth covers the 5-6 GHz band of WLAN 802.11a.
\end{abstract}

Index Terms-Dielectric resonators (DR), slot antennas.

\section{INTRODUCTION}

$\mathbf{T}$ HE loss of DR antennas at high frequency is lower than conventional patch antennas. Different approaches have been proposed to increase the impedance bandwidth of the former, for example, stacking multiple dielectric resonators with different resonant frequencies [1], inserting an air gap or a low-permittivity substrate between DR and the ground plane [2], [3].

Alternative approaches based on reducing the $Q$ factor of DRs have been proposed to incur a wider impedance bandwidth, for example, carving a notch [4], drilling a cavity off DR and fill it with lower-permittivity material [5], perforating holes in DR [6]. The impedance bandwidth can be increased by exciting different modes with close resonant bands [7]. DR antennas can also be combined with other types of antennas like patch or ring slot to extend their bandwidth [8], [9].

In this letter, a broadband DR antenna is proposed. A well is drilled off the DR to perturb the original electric field distribution of the $\mathrm{TE}_{111}^{y}$ mode, thus reducing its $Q$ factor. The effect of the well dimensions on the resonant frequency is studied. The coupling aperture also serves as a slot antenna, and its band is merged with that of the $\mathrm{TE}_{111}^{y}$ mode to achieve a wider bandwidth.

\section{Dielectric Resonator With a Well}

Fig. 1(a) shows a DR antenna with a well, fed by a microstrip line through aperture coupling. A rectilinear DR of dimensions $a \times b \times d$ is adhered to the ground plane, and a well of size $s_{1} \times s_{2} \times d$ is drilled off the DR with an offset $p$ between the well center and the DR center. The dimension of the coupling aperture is $L_{a} \times w_{a}$, and the microstrip line extends over the aperture by length $L_{s}$.

Manuscript received November 15, 2006; revised September 26, 2007. This work was supported by the National Science Council, Taiwan, ROC, under Contract NSC 93-2213-E-002-034.

The authors are with the Department of Electrical Engineering and the Graduate Institute of Communication Engineering, National Taiwan University, Taipei, Taiwan (e-mail: jfkiang@cc.ee.ntu.edu.tw).

Digital Object Identifier 10.1109/LAWP.2007.909963

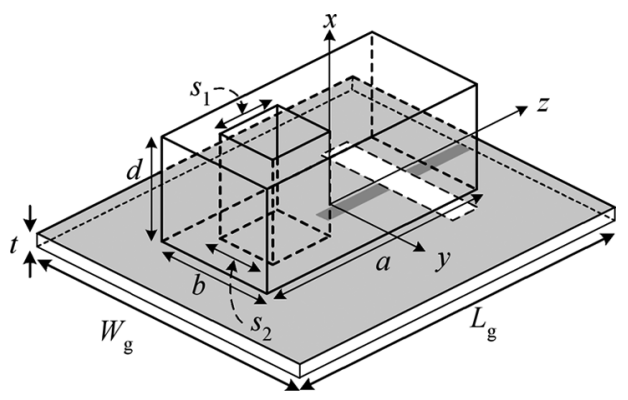

(a)

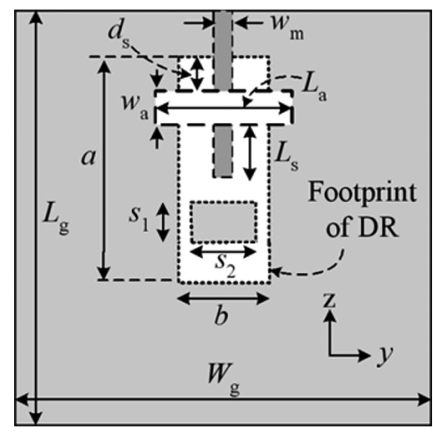

(b)

Fig. 1. Configuration of DR with an offset well: (a) schematic; (b) layout of feeding structure.

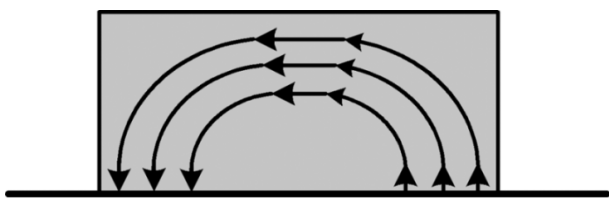

(a)

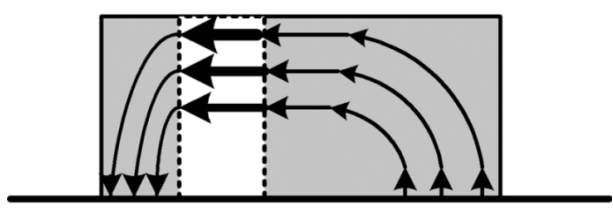

(b)

Fig. 2. Electric field distribution of $\mathrm{TE}_{111}^{y}$ mode in DR: (a) without well; (b) with a well.

Fig. 2 shows the electric field distribution of the $\mathrm{TE}_{111}^{y}$ mode within the rectilinear DR with or without the well. When a well is drilled off the DR, the electric field distribution is perturbed. Since the permittivity of the DR $\left(\epsilon_{r} \epsilon_{0}\right)$ is much larger than that of the air $\left(\epsilon_{0}\right)$, the electric field within the well is significantly enhanced, as depicted in Fig. 2(b), enabling more effective radiation. Hence, the $Q$ factor is decreased, and a wider bandwidth is obtained. 


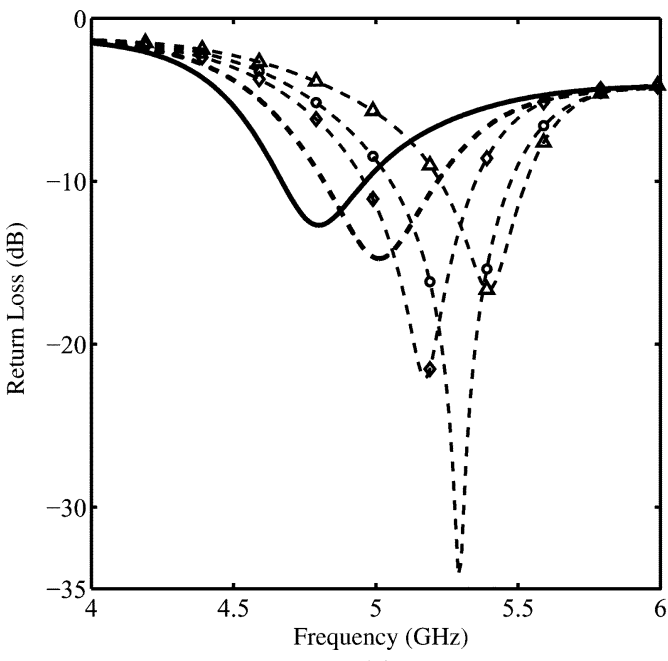

(a)

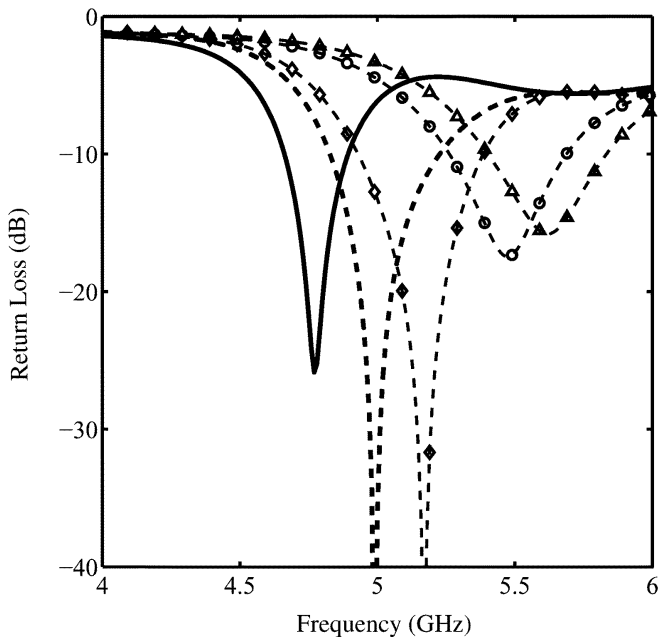

(b)

Fig. 3. Effects of well dimensions on the resonant frequency of $\mathrm{TE}_{111}^{y}$ mode, $a=15 \mathrm{~mm}, b=8 \mathrm{~mm}, d=6 \mathrm{~mm}, \epsilon_{r}=20, p=0 \mathrm{~mm}, w_{a}=1 \mathrm{~mm}$, $W_{g}=L_{g}=50 \mathrm{~mm}$. (a) Effect of $s_{2}, s_{1}=1 \mathrm{~mm}, d_{s}=4 \mathrm{~mm}, L_{s}=7 \mathrm{~mm}$, $L_{a}=8 \mathrm{~mm}$, 一: without well, - - -: $s_{2}=2 \mathrm{~mm},-\diamond-: s_{2}=3 \mathrm{~mm},-0-$ : $s_{2}=4 \mathrm{~mm},-\triangle-: s_{2}=5 \mathrm{~mm}$. (b) Effect of $s_{1}, s_{2}=5 \mathrm{~mm}, d_{s}=3 \mathrm{~mm}$, $L_{s}=6 \mathrm{~mm}, L_{a}=6.5 \mathrm{~mm},-: s_{1}=0.2 \mathrm{~mm},---: s_{1}=0.5 \mathrm{~mm},-\diamond-:$ $s_{1}=1 \mathrm{~mm},-0-: s_{1}=2 \mathrm{~mm},-\triangle-: s_{1}=3 \mathrm{~mm}$.

Fig. 3 shows the effect of well dimension on the resonant frequency. The 10-dB impedance bandwidth of the DR with a well is wider than that without well, and is increased as either $s_{1}$ or $s_{2}$ is increased. Note that the well dimensions do not affect the radiation patterns significantly.

Let $\left(\bar{E}_{0}, \bar{H}_{0}\right)$ and $(\bar{E}, \bar{H})$ be the field distributions of the $\mathrm{TE}_{111}^{y}$ mode without and with the well, respectively, $\omega_{0}$ and $\omega$ be the resonant frequencies of the $\mathrm{TE}_{111}^{y}$ mode without and with the well, respectively. The resonant frequency of the DR with well can be approximated as

$$
\omega=\frac{\tilde{W}_{m}+\tilde{W}_{e b}}{\tilde{W}_{m}+\tilde{W}_{e a}} \omega_{0}-\frac{j \iint_{S}\left(\bar{H} \times \bar{E}_{0}^{*}+\bar{H}_{0}^{*} \times \bar{E}\right) \cdot d \bar{s}}{\tilde{W}_{m}+\tilde{W}_{e a}}
$$

TABLE I

EFFECT OF WELL DiMENSIONS ON THE RESONANT FREQUENCY $f_{r}(\mathrm{GHz})$ AND THE BANDWIDTH BW (\%)

\begin{tabular}{lccccc}
\hline$s_{2}$ (mm) & 1 & 2 & 3 & 4 & 5 \\
$\Delta f_{r}$ (Eq. (1)) & 0.14 & 0.28 & 0.41 & 0.53 & 0.64 \\
$\Delta f_{r}$ BW (HFSS) & $0.13 / 6.7$ & $0.21 / 8$ & $0.37 / 7.5$ & $0.49 / 8$ & $0.6 / 5.5$ \\
\hline
\end{tabular}

Parameters (mm): $s_{1}=1, d_{s}=4, L_{a}=8, L_{s}=7$.

\begin{tabular}{lccccc}
\hline$s_{1}$ (mm) & 0.2 & 0.5 & 1 & 2 & 3 \\
$\Delta f_{r}$ (Eq. (1)) & 0.22 & 0.39 & 0.64 & 0.82 & 1.01 \\
$\Delta f_{r}$ /BW (HFSS) & $0.23 / 8$ & $0.4 / 8.8$ & $0.53 / 8.3$ & $0.7 / 7.8$ & $0.85 / 7.4$ \\
\hline
\end{tabular}

Parameters (mm): $s_{2}=6, d_{s}=3, L_{a}=6.5, L_{s}=6$.

Default (mm): $a=15, b=8, d=6, p=0, w_{a}=1$.

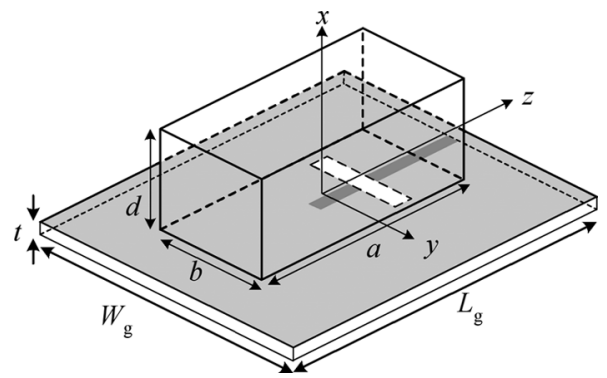

Fig. 4. Slot antenna with dielectric superstrate.

with

$$
\begin{aligned}
& \tilde{W}_{m}=\iiint_{V} \mu \bar{H}_{0}^{*} \cdot \bar{H} d v \\
& \tilde{W}_{e a}=\iiint_{V} \epsilon(\bar{r}) \bar{E} \cdot \bar{E}_{0}^{*} d v, \quad \tilde{W}_{e b}=\iiint_{V} \epsilon \bar{E}_{0}^{*} \cdot \bar{E} d v
\end{aligned}
$$

where $V$ is the volume of the DR without well, and $S$ is its surface. For example, an intact DR with dimensions $18 \mathrm{~mm} \times$ $8 \mathrm{~mm} \times 5 \mathrm{~mm}$ has the resonant frequency of $4.8 \mathrm{GHz}$ and the impedance bandwidth of about 5-6\%. The bandwidth can be increased to $6 \%-8.8 \%$ if a well is drilled off the DR.

Table I summarizes the effect of the well dimensions on the frequency shift caused by the well, where $\Delta f_{r}=\left(\omega-\omega_{0}\right) / 2 \pi$. Simulation results using HFSS are also compared.

\section{Slot Antenna With Dielectric Superstrate}

Fig. 4 shows a slot antenna covered with a finite-extent highpermittivity superstrate. The electric field gets stronger near the slot, and the width of superstrate $b$ has stronger effect on the resonant frequency than the length $a$. Fig. 5 shows the effect of superstrate thickness $d$ on the resonant frequency of the slot antenna. Larger value of $d$ implies higher effective permittivity or shorter wavelength as viewed by the slot, hence incurring a lower resonant frequency.

\section{RESULTS AND DiscusSIONS}

The input impedance can be matched by tuning the length of the extending microstrip line $L_{s}$, the aperture size $L_{a} \times w_{a}$, and the relative position between the DR and the aperture $d_{s}$. The well position $p$ is used to fine tune the resonant frequency and the input impedance. Fig. 6 shows the measured and simulated return loss of the DR with an offset well. The 10-dB impedance 


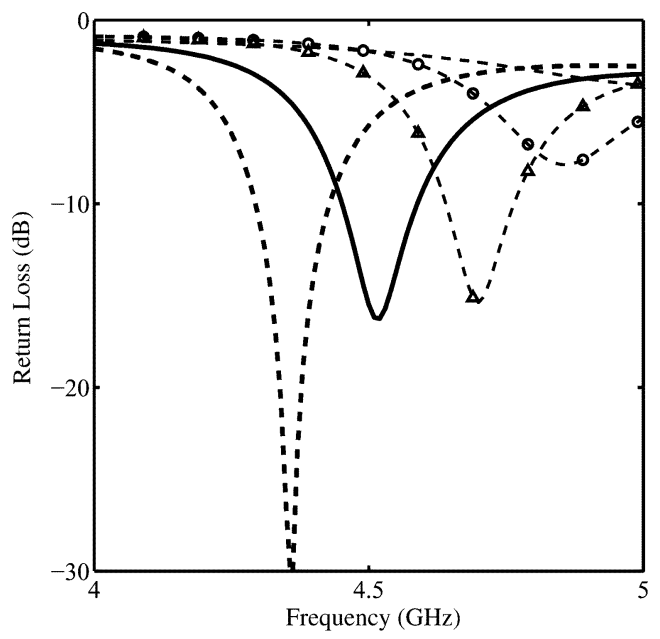

Fig. 5. Effect of superstrate thickness on the resonant frequency of slot antenna, $a=16 \mathrm{~mm}, b=8 \mathrm{~mm}, \epsilon_{r}=20, d_{s}=6 \mathrm{~mm}, w_{a}=1 \mathrm{~mm}, L_{a}=6 \mathrm{~mm}$, $L_{s}=3 \mathrm{~mm}, W_{g}=L_{g}=50 \mathrm{~mm},---: d=5.5 \mathrm{~mm},-: d=5 \mathrm{~mm},-\triangle-$ : $d=4.5 \mathrm{~mm},-\circ-: d=4 \mathrm{~mm}$.

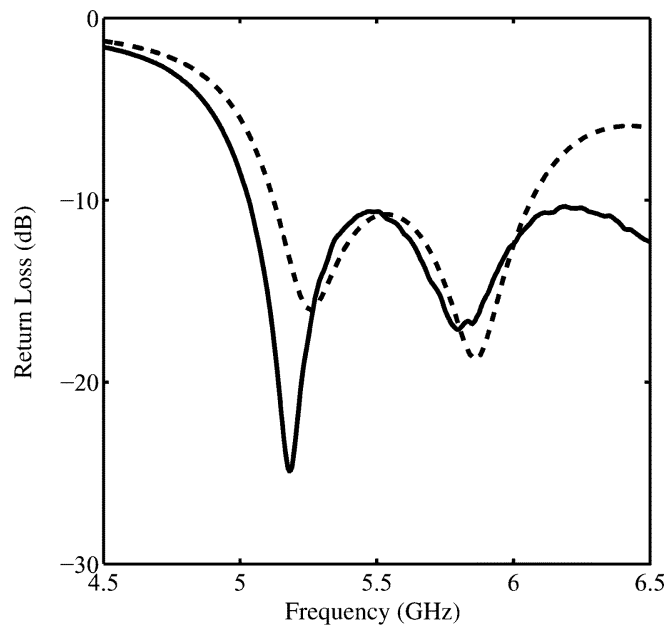

Fig. 6. Return loss of DRA with an offset well, - : measurement, - - -: simulation, $a=18.5 \mathrm{~mm}, b=8.1 \mathrm{~mm}, d=5 \mathrm{~mm}, s_{1}=5.6 \mathrm{~mm}, s_{2}=4.6 \mathrm{~mm}$, $p=2.45 \mathrm{~mm}, w_{a}=1 \mathrm{~mm}, d_{s}=1.7 \mathrm{~mm}, L_{a}=7 \mathrm{~mm}, L_{s}=2 \mathrm{~mm}$, $W_{g}=L_{g}=60 \mathrm{~mm}, t=0.6 \mathrm{~mm}, \epsilon_{r}=20$.

bandwidth is about $18 \%(5-6 \mathrm{GHz})$, which covers the WLAN $802.11 \mathrm{a}$ bands. The two nulls are associated with the slot antenna mode and the $\mathrm{TE}_{111}^{y}$ mode of the DR, respectively.

Fig. 7(a) shows the radiation pattern associated with the slot mode. The half-power beamwidth of the $E_{\theta}$ pattern is about $84^{\circ}\left(-42^{\circ} \leq \phi \leq 42^{\circ}\right)$, and the cross-polarization $\left(E_{\phi}\right)$ over the half-power beamwidth is $10 \mathrm{~dB}$ lower than the vertical polarization. The gain is $5.6 \mathrm{dBi}$ at $\phi=0^{\circ}$. Fig. 7(b) shows the radiation pattern associated with the $\mathrm{TE}_{111}^{y}$ mode of the DR. The half-power beamwidth of the $E_{\theta}$ pattern is about $94^{\circ}$ $\left(-48^{\circ} \leq \phi \leq 46^{\circ}\right)$, and the cross-polarization over the halfpower beamwidth is $12 \mathrm{~dB}$ lower than the vertical polarization. The gain is $4 \mathrm{dBi}$ at $\phi=0^{\circ}$. Note that the back radiation associated with the slot mode is stronger than that associated with the $\mathrm{TE}_{111}^{y}$ mode. For WLAN applications, for example, this DR antenna can be mounted on the wall with the $z$ axis pointing to ceiling, providing a broadside radiation pattern with vertical polarization on the $x y$-plane in front of the wall.

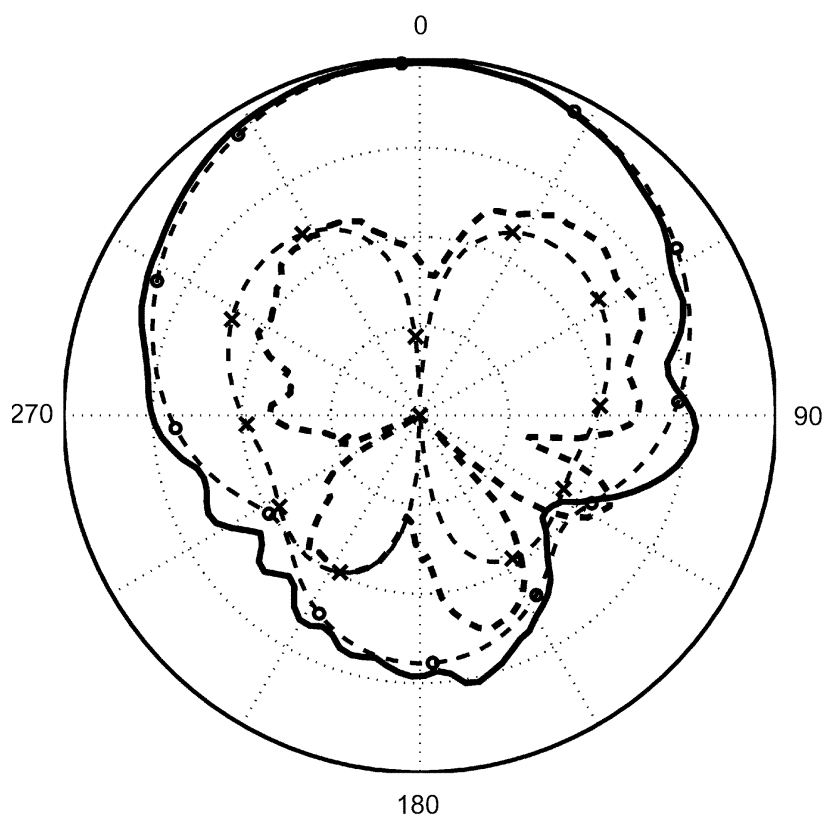

(a)

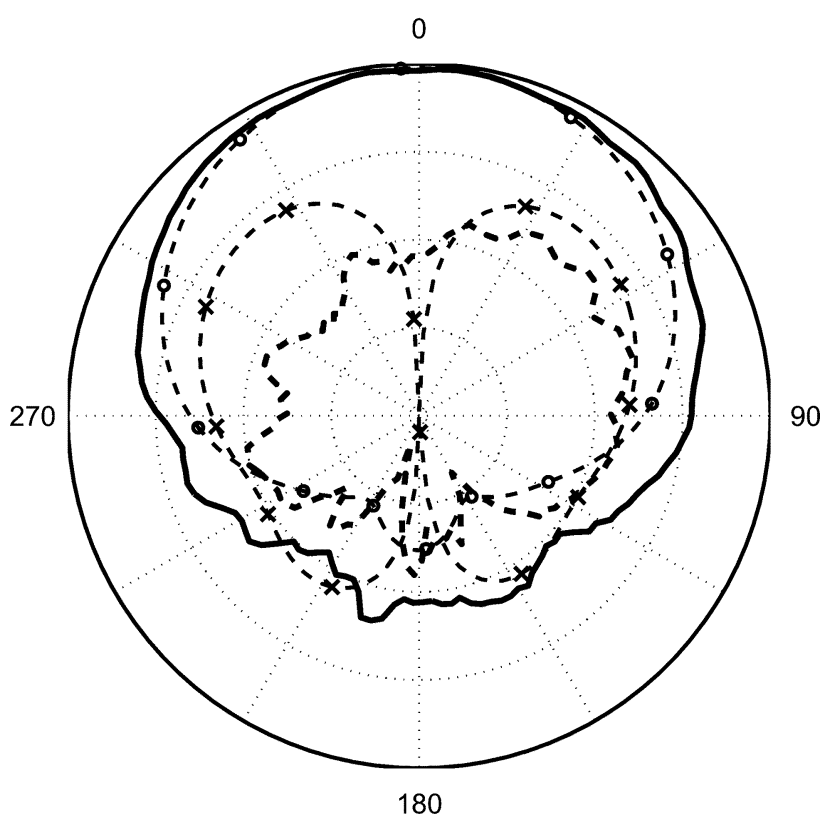

(b)

Fig. 7. Radiation patterns on the $x y$-plane at: (a) $f=5.2 \mathrm{GHz}$; (b) $f=$ $5.76 \mathrm{GHz}$, : measured $E_{\theta},---:$ measured $E_{\phi},-0-$ : simulated $E_{\theta},-\times-:$ simulated $E_{\phi}, 10-\mathrm{dB}$ per division on radials, all parameters are the same as in Fig. 6.

\section{CONCLUSION}

In this letter, the bandwidth of the $\mathrm{TE}_{111}^{y}$ mode of $\mathrm{DR}$ is increased by drilling a well, and is further extended by merging with the band of the slot mode, achieving a wider bandwidth of $18 \%(5-6 \mathrm{GHz})$. The $E_{\theta}$ pattern on the $x y$-plane has a halfpower beamwidth of about $90^{\circ}$ over the impedance bandwidth. Its gain is higher than $4 \mathrm{dBi}$. This antenna can be used in WLAN 802.11a applications.

\section{REFERENCES}

[1] A. A. Kishk, B. Ahn, and D. Kajfez, "Broadband stacked dielectric resonator antenna," Electron. Lett., vol. 25, no. 18, pp. 1232-1233, Aug. 1989. 
[2] S. M. Shum and K. M. Luk, "Characteristics of dielectric ring resonator antenna with an air gap," Electron. Lett., vol. 30, no. 4, pp. 277-278, Feb. 1994.

[3] A. Laisné, R. Gillard, and G. Piton, "Robust slot-fed dielectric resonator antenna using an intermediated substrate," Electron. Lett., vol. 37, no. 25, pp. 1497-1498, Dec. 2001.

[4] Y.-D. Kim, M.-S. Kim, and H.-M. Lee, "Internal rectangular dielectric resonator antenna with broadband characteristic for IMT-2000 handset," in Proc. IEEE APS Int. Symp., Jun. 2002, vol. 3, pp. 22-25.

[5] R. Chair, A. A. Kishk, and K. F. Lee, "Wideband low profile eye shaped dielectric resonator antennas," in Proc. IEEE APS Int. Symp., Jul. 2005, vol. 3A, pp. 582-585.
[6] R. Chair, A. A. Kishk, and K. F. Lee, "Experimental investigation for wideband perforated dielectric resonator antenna," Electron. Lett., vol. 42, no. 3, pp. 1497-1498, Feb. 2006

[7] A. A. Kishk, A. W. Glisson, and G. P. Junker, "Bandwidth enhancement for split cylindrical dielectric resonator antennas," Progress in Electromagn. Res., vol. 33, pp. 97-118, 2001.

[8] K. P. Esselle and T. S. Bird, "A hybrid-resonator antenna: Experimental results," IEEE Trans. Antennas Propag., vol. 53, pp. 870-871, Feb. 2005.

[9] T. A. Denidni and Q. Rao, "Hybrid dielectric resonator antennas with radiating slot for dual-frequency operation," IEEE Antennas Wireless Propag., vol. 3, pp. 321-323, 2004. 